\title{
The Bifurcation of Cycle Length and Global Asymptotic Stability in a Rational Difference Equation with Higher Order
}

\author{
Dongmei Chen and Xianyi Li*
}

College of Mathematics and Computational Science,Shenzhen University, Shenzhen, Guangdong 518060, P. R. China

Abstract: A new bifurcation case for the cycle length is found in this paper for rational difference equations, which is shown out from the following fifth order rational difference equation

$$
x_{n+1}=\frac{x_{n-1} x_{n-2}+x_{n-1} x_{n-4}+x_{n-2} x_{n-4}+1+a}{x_{n-1} x_{n-2} x_{n-4}+x_{n-1}+x_{n-2}+x_{n-4}+a}, n=0,1,2, \cdots,
$$

where $a \in[0, \infty)$ and the initial values $x_{-4}, x_{-3}, x_{-2}, x_{-1}, x_{0} \in(0, \infty)$. Mainly, the perturbation of the initial values may lead to the essential variation of the cycle length rule for the nontrivial solutions of the equation. That is, with the change of the initial values, the successive lengths of positive and negative semicycles for nontrivial solutions of this equation is found to periodically occur with multiple different prime periods, respectively, 4,12 . Furthermore, in any one fixed period, the successive occurring order of positive and negative semicycles is completely inverse, i.e., for the period 4 , the order is either $3^{+}, 1^{-}$or $3^{-}, 1^{+}$in a period, and for the period 12 , the order is either $5^{+}, 2^{-}, 1^{+}, 1^{-}, 1^{+}, 2^{-}$or $5^{-}, 2^{+}, 1^{+}, 1^{-}, 1^{+}, 2^{+}$in a period. This rule is different from the known one we have obtained for various rational difference equations. By the use of the rule its positive equilibrium point is verified to be globally asymptotically stable.

Keywords: Rational difference equation, bifurcation of cycle length, perturbation, global asymptotic stability, semicycle, periodicity.

\section{INTRODUCTION}

The qualitative properties of rational difference equations have been the object studied in the recent years. For the systematical investigation of rational difference equations, one can refer to the monographs $[1,2]$ and [3-10], especially, the papers published in the two international journals "Advances in Difference Equations" and "Journal of Difference Equations and Applications" and the references cited therein.

Generally speaking, given a difference equation

$$
x_{n+1}=f\left(x_{n}, \mu\right), n=0,1,2, \cdots,
$$

where $x_{n} \in R^{m}, \mu \in R^{k}, f \in C\left(R^{m+k}, R^{m}\right), m, k \in\{1,2, \cdots\}$ and the initial value $x_{0} \in R^{m}$, its solution is a continuous function with respect to the initial value $x_{0}$ and the parameter $\mu$, denoted by $x_{n}=x\left(n, x_{0}, \mu\right)$. If the change of the initial value $x_{0}$ or the parameter $\mu$ around a value leads to the essential change of the trajectory structure rule of its solution, then it is called that a bifurcation of this equation occurs.

*Address correspondence to this author at the College of Mathematics and Computational Science, Shenzhen University, Shenzhen, Guangdong 518060, P. R. China; E-mail: cddmmm@szu.edu.cn, xyli@szu.edu.cn
Correspondingly, the critical value is called to be a bifurcation value. This is similar to the definition of the bifurcation of ordinary differential equation.

Certainly, it should be pointed out that the essential change of the trajectory structure rule of a difference equation contains many cases, such as, a solution from the stability to the unstability, from the boundedness to the unboundedness, from one period to another period, or the cycle length from one period to another period, etc. Therefore, it is meaningful to investigate the bifurcation theory of difference equation according to its own right.

In this paper, we study the rule of cycle length and global asymptotic stability of the fifth order rational difference equation

$$
x_{n+1}=\frac{x_{n-1} x_{n-2}+x_{n-1} x_{n-4}+x_{n-2} x_{n-4}+1+a}{x_{n-1} x_{n-2} x_{n-4}+x_{n-1}+x_{n-2}+x_{n-4}+a}, n=0,1,2, \cdots
$$

where $a \in[0, \infty)$ and the initial values $x_{-4}, x_{-3}, x_{-2}, x_{-1}$, $x_{0} \in(0, \infty)$.

According to our recent work [6-10], some bifurcation cases are found for the lengths of positive and negative semicycles of nontrivial solutions of some rational difference equations to successively occur. The rule, generally 
speaking, is that the length of semicycle occurs periodically with the following three cases.

(i) The period is fixed; and, moreover, in the fixed period, the order for the lengths of positive and negative semicycles to successively occur is also fixed $[6,7,8]$;

(ii) The period is fixed; however, in the fixed period, the order for the lengths of positive and negative semicycles to successively occur is changeable [9];

(iii) The period is changeable, i,e,. possessing multi-different prime periods; whereas, in any one period, the order for the lengths of positive and negative semicycles to successively occur is fixed [10].

In this note, we find a new case for the rule of the cycle length, which is also our main aim to write this paper for Eq.(1), that is the following case (iv).

(iv) The period is changeable, i,e,. possessing different periods; Furthermore, in any one period, the order for the lengths of positive and negative semicycles to successively occur is also changeable, furthermore completely inverse.

Mainly we find the following bifurcation phenomenon: if we fix some four of the five initial values $x_{-4}, x_{-3}, x_{-2}, x_{-1}, x_{0} \in(0.1) \cup(1, \infty)$ and let the residual one perturb around the equilibrium point of equation (1), then the essential variation will take place for the rule for the trajectory structure of solutions of the equation. In detail, with the perturbation of the initial values, the successive length of positive and negative semicycles of nontrivial solutions of equation (1) is found to periodically occur with multiple different prime periods, respectively, 4,12 . Furthermore, in any one fixed period, the successive occuring order of positive and negative semicycles is completely inverse, i.e., for the period 4 , the order is either $\cdots, 3^{+}, 1^{-}, 3^{+}, 1^{-}, 3^{+}, 1^{-} 3^{+}, 1^{-}, \cdots$ or $\cdots, 3^{-}, 1^{+}, 3^{-}, 1^{+}, 3^{-}, 1^{+}, 3^{-}, 1^{+}, \cdots$, and for the period 12 , the order is either $5^{+}, 2^{-}, 1^{+}, 1^{-}, 1^{+}, 2^{-}$or $5^{-}, 2^{+}, 1^{+}, 1^{-}, 1^{+}, 2^{+}$ in a period. By utilizing the rule, we show the positive equilibrium point of equation (1) to be globally asymptotically stable.

The positive equilibrium $\bar{x}$ of equation (1) satisfies $\bar{x}=\frac{3 \bar{x}^{2}+1+a}{\bar{x}^{3}+3 \bar{x}+a}$, which shows $\bar{x}=1$.

The following definitions will be used in this paper.

\section{DEFINITION 1.1.}

A positive semicycle of a solution $\left\{x_{n}\right\}_{n=-4}^{\infty}$ of equation (1) consists of a"string"of terms $\left\{x_{l}, x_{l+1}, \cdots, x_{m}\right\}$, all greater than or equal to the equilibrium $\bar{x}$, with $l \geq-4$ and $m \leq \infty$ such that

either $l=-4$ or $l>-4$ and $x_{l-1}<\bar{x}$

and

either $m=\infty$ or $m<\infty$ and $x_{m+1}<\bar{x}$.
A negative semicycle of a solution $\left\{x_{n}\right\}_{n=-4}^{\infty}$ of equation (1) consists of a"string"of terms $\left\{x_{l}, x_{l+1}, \cdots, x_{m}\right\}$, all less than $\bar{x}$, with $l \geq-4$ and $m \leq \infty$ such that either $l=-4$ or $l>-4$ and $x_{l-1} \geq \bar{x}$

and

either $m=\infty$ or $m<\infty$ and $x_{m+1} \geq \bar{x}$.

The length of a semicycle is the number of the total terms contained in it.

\section{DEFINITION 1.2.}

A solution $\left\{x_{n}\right\}_{n=-4}^{\infty}$ of equation (1) is said to be eventually trivial if $x_{n}$ is eventually equal to $\bar{x}=1$; Otherwise, the solution is said to be nontrivial.

For the other concepts in this paper, see $[1,2]$.

\section{TWO LEMMAS}

We first establish two lemmas which will play a key role in the proof of main results in the sequel.

\section{LEMMA 2.1.}

A positive solution $\left\{x_{n}\right\}_{n=-4}^{\infty}$ of equation (1) is eventually equal to 1 if and only if

$\left(x_{-4}-1\right)\left(x_{-3}-1\right)\left(x_{-2}-1\right)\left(x_{-1}-1\right)\left(x_{0}-1\right)=0$.

Proof. Assume that (2) holds. Then according to equation (1), it is easy to see that the following conclusions hold.

i) If $x_{-4}=1$, then $x_{n}=1$ for $n \geq 3$;

ii) If $x_{-3}=1$, then $x_{n}=1$ for $n \geq 4$;

iii) If $x_{-2}=1$, then $x_{n}=1$ for $n \geq 3$;

iv) If $x_{-1}=1$, then $x_{n}=1$ for $n \geq 1$;

v) If $x_{0}=1$, then $x_{n}=1$ for $n \geq 2$.

Conversely, assume that

$\left(x_{-4}-1\right)\left(x_{-3}-1\right)\left(x_{-2}-1\right)\left(x_{-1}-1\right)\left(x_{0}-1\right) \neq 0$.

Then one can show that

$x_{n} \neq 1$ for any $n \geq 1$.

Assume the contrary that for some $N \geq 1$,

$x_{N}=1$ and that $x_{n} \neq 1$ for $-4 \leq n \leq N-1$. (4)

It is easy to see that

$1=x_{N}=\frac{x_{N-2} x_{N-3}+x_{N-2} x_{N-5}+x_{N-3} x_{N-5}+1+a}{x_{N-2} x_{N-3} x_{N-5}+x_{N-2}+x_{N-3}+x_{N-5}+a}$,

which implies

$\left(x_{N-2}-1\right)\left(x_{N-3}-1\right)\left(x_{N-5}-1\right)=0$.

Obviously, this contradicts (4). 


\section{REMARK 2.1.}

If the initial conditions do not satisfy equality (2), then, for any solution $\left\{x_{n}\right\}$ of equation (1), $x_{n} \neq 1$ for $n \geq-4$. Hence, the solution is actually a nontrivial one.

\section{LEMMA 2.2.}

Let $\left\{x_{n}\right\}_{n=-4}^{\infty}$ be a nontrivial positive solution of equation (1). Then the following conclusions are true:

(a) $\left(x_{n+1}-1\right)\left(x_{n-1}-1\right)\left(x_{n-2}-1\right)\left(x_{n-4}-1\right)<0$ for $n \geq 0$;

(b) $\left(x_{n+1}-x_{n-1}\right)\left(x_{n-1}-1\right)<0$ for $n \geq 0$;

(c) $\left(x_{n+1}-x_{n-2}\right)\left(x_{n-2}-1\right)<0$ for $n \geq 0$;

(d) $\left(x_{n+1}-x_{n-4}\right)\left(x_{n-4}-1\right)<0$ for $n \geq 0$.

Proof In view of equation (1), we have that

$$
x_{n+1}-1=-\frac{\left(x_{n-1}-1\right)\left(x_{n-2}-1\right)\left(x_{n-4}-1\right)}{x_{n-1} x_{n-2} x_{n-4}+x_{n-1}+x_{n-2}+x_{n-4}+1+a},
$$

$n=0,1,2, \cdots$ and

$x_{n+1}-x_{n-1}=\frac{\left(1-x_{n-1}\right)\left[x_{n-2} x_{n-4}\left(1+x_{n-1}\right)+\left(1+x_{n-1}\right)+a\right]}{x_{n-1} x_{n-2} x_{n-4}+x_{n-1}+x_{n-2}+x_{n-4}+1+a}$,

$n=0,1,2, \cdots$, from which inequalities (a) and (b) follow. The proofs for inequalities (c) and (d) are similar to the one for inequality (b).

\section{MAIN RESULTS AND THEIR PROOFS}

In this section, we mainly investigate the properties of nontrivial solutions of equation (1). First, we study the oscillation of nontrivial solutions of equation (1). We have the result as follows.

\section{THEOREM 3.1.}

Any one nontrivial solution of equation (1) is strictly oscillatory. Furthermore, regardless of positive semicycle or negative semicycle, the cycle length is at most five.

Proof Let $\left\{x_{n}\right\}_{n=-4}^{\infty}$ be any one nontrivial solution of equation (1). It follows from Remark 2.1 that $x_{n} \neq 1$ for any $n \geq-4$. For some nonnegative intege $p$, if $x_{p-4}>1, x_{p-3}>1$, $x_{p-2}>1, x_{p-1}>1$ and $x_{p}>1$, then, from Lemma 2.2 (a), one can see $x_{p+1}<1$. Conversely, if $x_{p-4}<1, x_{p-3}<1, x_{p-2}<1$, $x_{p-1}<1$ and $x_{p}<1$, then, Lemma 2.2 (a) implies $x_{p+1}>1$. So, from those, we know the conclusion of Theorem 3.1 is true.

Theorem 3.1 tells us that every nontrivial solution of equation (1) is strictly oscillatory. Then, a problem naturally rises: How does equation (1) oscillate ? This need further studying the rule of the cycle length of equation (1). Therefore, next, we consider the rule for the cycle lengths of positive and negative semeicycles of nontrivial solutions of equation (1) to occur successively.

\section{THEOREM 3.2.}

Let $\left\{x_{n}\right\}_{n=-4}^{\infty}$ be a nontrivial solution of equation (1). Then, the bifurcation of cycle length with respect to the initial values happens and the bifurcations value is $\left(x_{-4}, x_{-3}, x_{-2}, x_{-1}, x_{0}\right)=(1,1,1,1,1)$. Namely, with the perturbation of the initial values, the successive lengths of positive and negative semicycles of the solution will periodically occur with multi-different prime periods, respectively, 4, 12. Furthermore, in any one of the fixed periods, the successive order of positive and negative semicycles is completely converse. In detail, for the period 4, the order is either $\cdots, 3^{+}, 1^{-}, 3^{+}, 1^{-}, 3^{+}, 1^{-}, 3^{+}, 1^{-}, \cdots$ or $\cdots, 3^{-}, 1^{+}, 3^{-}, 1^{+}, 3^{-}, 1^{+}, 3^{-}, 1^{+}, \cdots$, and for the period 12 , the order is either $5^{+}, 2^{-}, 1^{+}, 1^{-}, 1^{+}, 2^{-}$or $5^{-}, 2^{+}, 1^{+}, 1^{-}$, $1^{+}, 2^{+}$in a period.

Proof It follows from Theorem 3.1 that every nontrivial solution of equation (1) is strictly oscillatory and the length of a negative semicycle or a positive semicycle is at most 5 . From remark 2.1, we see that the initial values satisfy

$\left(x_{-4}-1\right)\left(x_{-3}-1\right)\left(x_{-2}-1\right)\left(x_{-1}-1\right)\left(x_{0}-1\right) \neq 0$,

which leads to that, for some integer $p \geq 0$, one of the following eight cases must occur:

Case 1: $x_{p-4}>1, x_{p-3}<1, x_{p-2}>1, x_{p-1}>1$ and $x_{p}>1$;

Case 2: $x_{p-4}>1, x_{p-3}<1, x_{p-2}>1, x_{p-1}>1$ and $x_{p}<1$;

Case 3: $x_{p-4}>1, x_{p-3}<1, x_{p-2}>1, x_{p-1}<1$ and $x_{p}>1$;

Case 4: $x_{p-4}>1, x_{p-3}<1, x_{p-2}>1, x_{p-1}<1$ and $x_{p}<1$;

Case 5: $x_{p-4}>1, x_{p-3}<1, x_{p-2}<1, x_{p-1}>1$ and $x_{p}>1$;

Case 6: $x_{p-4}>1, x_{p-3}<1, x_{p-2}<1, x_{p-1}>1$ and $x_{p}<1$;

Case 7: $x_{p-4}>1, x_{p-3}<1, x_{p-2}<1, x_{p-1}<1$ and $x_{p}>1$;

Case 8: $x_{p-4}>1, x_{p-3}<1, x_{p-2}<1, x_{p-1}<1$ and $x_{p}<1$.

If Case 1 occurs, it follows from Lemma 2.2 (a) that $x_{p+1}<1, x_{p+2}>1, x_{p+3}>1, x_{p+4}>1, x_{p+5}<1, x_{p+6}>1$, $x_{p+7}>1, x_{p+8}>1, x_{p+9}<1, x_{p+10}>1, x_{p+11}>1, x_{p+12}>1$, $x_{p+13}<1, x_{p+14}>1, x_{p+15}>1, x_{p+16}>1, x_{p+17}<1, x_{p+18}>1$, $x_{p+19}>1, x_{p+20}>1, x_{p+21}<1, x_{p+22}>1, x_{p+23}>1, x_{p+24}>1$, $x_{p+25}<1, x_{p+26}>1, x_{p+27}>1, x_{p+28}>1, x_{p+29}<1, \cdots$. This shows that the rule for the lengths of positive and negative semicycles of the solution of equation (1) to successively occur is $\cdots, 3^{+}, 1^{-}, 3^{+}, 1^{-}, 3^{+}, 1^{-}, 3^{+}, 1^{-}, 3^{+}, 1^{-} 3^{+}, 1^{-}, \cdots$.

If Case 2 comes up, then Lemma 2.2 (a) implies that $x_{p+1}<1, x_{p+2}<1, x_{p+3}<1, x_{p+4}<1, x_{p+5}>1, x_{p+6}>1$, $x_{p+7}<1, x_{p+8}>1, x_{p+9}<1, x_{p+10}>1, x_{p+11}>1, x_{p+12}<1$, $x_{p+13}<1, x_{p+14}<1, x_{p+15}<1, x_{p+16}<1, x_{p+17}>1, x_{p+18}>1$, $x_{p+19}<1, x_{p+20}>1, x_{p+21}<1, x_{p+22}>1, x_{p+23}>1, x_{p+24}<1$, $x_{p+25}<1, x_{p+26}<1, x_{p+27}<1, x_{p+28}<1, x_{p+29}>1, x_{p+30}>1$, 
$x_{p+31}<1, x_{p+32}>1, x_{p+33}<1, x_{p+34}>1, x_{p+35}>1, \cdots$, which indicates the rule for the numbers of terms of positive and negative semicycles of the solution of equation (1) to successively occur is $\cdots, 5^{-}, 2^{+}, 1^{-}, 1^{+}, 1^{-}, 2^{+}, 5^{-}, 2^{+}$, $1^{-}, 1^{+}, 1^{-}, 2^{+}, 5^{-}, 2^{+}, 1^{-}, 1^{+}, 1^{-}, 2^{+}, \cdots$.

When Case 3 happens, a similar deduction leads to that $x_{p+1}>1, x_{p+2}<1, x_{p+3}<1, x_{p+4}<1, x_{p+5}<1, x_{p+6}<1$, $x_{p+7}>1, x_{p+8}>1, x_{p+9}<1, x_{p+10}>1, x_{p+11}<1, x_{p+12}>1$, $x_{p+13}>1, x_{p+14}<1, x_{p+15}<1, x_{p+16}<1, x_{p+17}<1, x_{p+18}<1$, $x_{p+19}>1, x_{p+20}>1, x_{p+21}<1, x_{p+22}>1, x_{p+23}<1$, $x_{p+24}>1, x_{p+25}>1, x_{p+26}<1, x_{p+27}<1, x_{p+28}<1, x_{p+29}<1$, $x_{p+30}<1, x_{p+31}>1, x_{p+32}>1, x_{p+33}<1, x_{p+34}>1, x_{p+35}<1$, $x_{p+36}>1, x_{p+37}>1, \cdots$ which displays the rule for the numbers of terms of positive and negative semicycles of the solution of equation (1) to successively occur is $\cdots, 5^{-}, 2^{+}$, $1^{-}, 1^{+}, 1^{-}, 2^{+}, 5^{-}, 2^{+}, 1^{-}, 1^{+}, 1^{-}, 2^{+}, 5^{-}, 2^{+}, 1^{-}, 1^{+}$, $1^{-}, 2^{+}, \cdots$.

If Case 4 arrives at, then Lemma 2.2 (a) tells us that $x_{p+1}>1, x_{p+2}>1, x_{p+3}>1, x_{p+4}>1, x_{p+5}>1, x_{p+6}<1$, $x_{p+7}<1, x_{p+8}>1, x_{p+9}<1, x_{p+10}>1, x_{p+11}<1, x_{p+12}<1$, $x_{p+13}>1, x_{p+14}>1, x_{p+15}>1, x_{p+16}>1, x_{p+17}>1, x_{p+18}<1$, $x_{p+19}<1, x_{p+20}>1, x_{p+21}<1, x_{p+22}>1, x_{p+23}<1$, $x_{p+24}<1, x_{p+25}>1, x_{p+26}>1, x_{p+27}>1, x_{p+28}>1, x_{p+29}>1$, $x_{p+30}<1, x_{p+31}<1, x_{p+32}>1, x_{p+33}<1, x_{p+34}>1, x_{p+35}<1$, $x_{p+36}<1, \cdots$. This shows the rule for the cycle length of the solution of equation (1) to successively occur is $\cdots, 5^{+}, 2^{-}$, $1^{+}, 1^{-}, 1^{+}, 2^{-}, 5^{+}, 2^{-}, 1^{+}, 1^{-}, 1^{+}, 2^{-}, 5^{+}, 2^{-}, 1^{+}, 1^{-}$, $1^{+}, 2^{-}, \cdots$.

If Case 5 occurs, it follows still from Lemma 2.2 (a) that $x_{p+1}>1, x_{p+2}>1, x_{p+3}>1, x_{p+4}<1, x_{p+5}<1, x_{p+6}>1$, $x_{p+7}<1, x_{p+8}>1, x_{p+9}<1, x_{p+10}<1, x_{p+11}>1, x_{p+12}>1$, $x_{p+13}>1, x_{p+14}>1, x_{p+15}>1, x_{p+16}<1, x_{p+17}<1, x_{p+18}>1$, $x_{p+19}<1, x_{p+20}>1, x_{p+21}<1, x_{p+22}<1, x_{p+23}>1, x_{p+24}>1$, $x_{p+25}>1, x_{p+26}>1, x_{p+27}>1, x_{p+28}<1, x_{p+29}<1, x_{p+30}>1$, $x_{p+31}<1, x_{p+32}>1, x_{p+33}<1, x_{p+34}<1, x_{p+35}>1, x_{p+36}>1$, $x_{p+37}>1, x_{p+38}>1, x_{p+39}>1, x_{p+40}<1, x_{p+41}<1, x_{p+42}>1$, $x_{p+43}<1, x_{p+44}>1, x_{p+45}<1, x_{p+46}<1, \cdots$, which manifests the rule for the cycle length of the solution is still $\cdots, 5^{+}, 2^{-}$, $1^{+}, 1^{-}, 1^{+}, 2^{-}, 5^{+}, 2^{-}, 1^{+}, 1^{-}, 1^{+}, 2^{-}, 5^{+}, 2^{-}, 1^{+}, 1^{-}$, $1^{+}, 2^{-}, \cdots$.

When Case 6 appears, a similar deduction gives rise to that $x_{p+1}>1, x_{p+2}<1, x_{p+3}<1, x_{p+4}>1$, $x_{p+5}>1, x_{p+6}>1, x_{p+7}>1, x_{p+8}>1, x_{p+9}<1, x_{p+10}<1$, $x_{p+11}>1, x_{p+12}<1, x_{p+13}>1, x_{p+14}<1, x_{p+15}<1$, $x_{p+16}>1, x_{p+17}>1, x_{p+18}>1, x_{p+19}>1, x_{p+20}>1$, $x_{p+21}<1, \quad x_{p+22}<1, \quad x_{p+23}>1, \quad x_{p+24}<1$, $x_{p+25}>1, x_{p+26}<1, x_{p+27}<1, \cdots$, which displays that the regulation for the lengths of positive and negative semicycles which occur successively is $\cdots, 5^{+}, 2^{-}, 1^{+}, 1^{-}, 1^{+}, 2^{-}, 5^{+}$, $2^{-}, 1^{+}, 1^{-}, 1^{+}, 2^{-}, 5^{+}, 2^{-}, 1^{+}, 1^{-}, 1^{+}, 2^{-}, \cdots$.

When Case 7 occurs, one can see from Lemma 2.2 (a) that $x_{p+1}<1, x_{p+2}<1, x_{p+3}<1, x_{p+4}>1, x_{p+5}<1, x_{p+6}<1$, $x_{p+7}<1, x_{p+8}>1, x_{p+9}<1, x_{p+10}<1, x_{p+11}<1, x_{p+12}>1$, $x_{p+13}<1, x_{p+14}<1, x_{p+15}<1, x_{p+16}>1, x_{p+17}<1, x_{p+18}>1$, $x_{p+19}<1, x_{p+20}>1, x_{p+21}<1, x_{p+22}<1, x_{p+23}<1$, $x_{p+24}>1, \cdots$.This proves the rule for the numbers of terms of positive and negative semicycles of the solution of equation (1) to successively occur still is $\cdots, 3^{-}, 1^{+}, 3^{-}, 1^{+}, 3^{-}, 1^{+}$, $3^{-}, 1^{+}, 3^{-}, 1^{+}, \cdots$.

When Case 8 appears, similarly, according to Lemma 2.2 (a), we obtain that $x_{p+1}<1, x_{p+2}>1, x_{p+3}>1, x_{p+4}<1$, $x_{p+5}>1, x_{p+6}<1, x_{p+7}>1, x_{p+8}>1, x_{p+9}<1, x_{p+10}<1$, $x_{p+11}<1, x_{p+12}<1, x_{p+13}<1, x_{p+14}>1, x_{p+15}>1, x_{p+16}<1$, $x_{p+17}>1, x_{p+18}<1, x_{p+19}>1, x_{p+20}>1, x_{p+21}<1, x_{p+22}<1$, $x_{p+23}<1, x_{p+24}<1, x_{p+25}<1, x_{p+26}>1, x_{p+27}>1, x_{p+28}<1$, $x_{p+29}>1, x_{p+30}<1, x_{p+31}>1, x_{p+32}>1, x_{p+33}<1, x_{p+34}<1$, $x_{p+35}<1, x_{p+36}<1, x_{p+37}<1, x_{p+38}>1, x_{p+39}>1, x_{p+40}<1$, $x_{p+41}>1, x_{p+42}<1, \cdots$, which proves that the rule for the lengths of positive and negative semicycles which occur successively is $\cdots, 5^{+}, 2^{-}, 1^{+}, 1^{-}, 1^{+}, 2^{-}, 5^{+}, 2^{-}, 1^{+}, 1^{-}$, $1^{+}, 2^{-}, 5^{+}, 2^{-}, 1^{+}, 1^{-}, 1^{+}, 2^{-}, \cdots$.

Therefore, the proof is complete.

Since we have been clear for the rule for the cycle length of trajectory structure of nontrivial solution of equation (1), we'd like to know whether the unique positive equilibrium of equation(1) is globally asymptotically stable. The question is positively answered in the following, which is the third main result in this note.

\section{THEOREM 3.3.}

Assume that $a \in[0, \infty)$. Then the positive equilibrium of equation (1) is globally asymptotically stable.

Proof We must prove that the positive equilibrium point $\bar{x}$ of equation (1) is both locally asymptotically stable and globally attractive. The linearized equation of equation (1) about the positive equilibrium $\bar{x}=1$ is

$y_{n+1}=0 \cdot y_{n}+0 \cdot y_{n-1}+0 \cdot y_{n-2}+0 \cdot y_{n-3}+0 \cdot y_{n-4}, n=0,1, \cdots$.

By virtue of [2, Remark 1. 3. 1], $\bar{x}$ is locally asymptotically stable. It remains to verify that every positive solution $\left\{x_{n}\right\}_{n=-4}^{\infty}$ of equation (1) converges to 1 as $n \rightarrow \infty$. Namely, we want to prove

$\lim _{n \rightarrow \infty} x_{n}=\bar{x}=1$. 
If the initial values of the solution satisfy (2), then Lemma 1 says the solution is eventually equal to 1 (i.e., trivial solution) and of course, (5) holds. Therefore, we assume in the following that the initial values of the solution do not satisfy (2). Then, by remark 2.1 , we know, for any solution $\left\{x_{n}\right\}$ of equation (1), $x_{n} \neq 1$ for $n \geq-4$ (i.e., nontrivial solution).

Now, let $\left\{x_{n}\right\}$ be a nontrivial solution of equation (1). In view of Theorem 3.2, one can see that the successive lengths of positive and negative semicycles occur periodically with different prime periods, respectively, 4,12 . In a period, the rule is

(C1) $5^{+}, 2^{-}, 1^{+}, 1^{-}, 1^{+}, 2^{-}$, or

(C2) $5^{-}, 2^{+}, 1^{-}, 1^{+}, 1^{-}, 2^{+}$, or

(C3) $3^{+}, 1^{-}$, or

(C4) $3^{-}, 1^{+}$.

Cases $(\mathrm{C} 1)$ and $(\mathrm{C} 2),(\mathrm{C} 3)$ and $(\mathrm{C} 4)$ are respectively symmetrical. It suffices to consider the cases (C1) and (C3). First, we consider the case (C1). For simplicity, for some nonnegative integer $p$, we denote by $\left\{x_{p}, x_{p+1}, x_{p+2}, x_{p+3}, x_{p+4}\right\}^{+}$the terms of a positive semicycle of length five, followed by $\left\{x_{p+5}, x_{p+6}\right\}^{-}$a negative semicycle with length two, then a positive semicycle $\left\{x_{p+7}\right\}^{+}$, a negative semicycle $\left\{x_{p+8}\right\}^{-}$, a positive semicycle $\left\{x_{p+9}\right\}^{+}$, a negative semicycle $\left\{x_{p+10}, x_{p+11}\right\}^{-}$, and so on. Namely, the rule for the positive and negative semicycles to occur successively can be periodically expressed as follows:

$$
\begin{aligned}
& \left\{x_{p+12 n}, x_{p+12 n+1}, x_{p+12 n+2}, x_{p+12 n+3}, x_{p+12 n+4}\right\}^{+}, \\
& \left\{x_{p+12 n+5}, x_{p+12 n+6}\right\}^{-},\left\{x_{p+12 n+7}\right\}^{+},\left\{x_{p+12 n+8}\right\}^{-},\left\{x_{p+12 n+9}\right\}^{+}, \\
& \left\{x_{p+12 n+10}, x_{p+12 n+1}\right\}^{-}, n=0,1, \cdots .
\end{aligned}
$$

From Lemma 2.2 (b) and (c), respectively, we may straightforwardly obtain the following results:

(i) $x_{p+12 n+21}<x_{p+12 n+19}<x_{p+12 n+16}<x_{p+12 n+14}<x_{p+12 n+12}$

$<x_{p+12 n+9}<x_{p+12 n+7}<x_{p+12 n+4}<x_{P+12 n+2}<x_{p+12 n} ;$

(ii) $x_{p+12 n+22}>x_{p+12 n+20}>x_{p+12 n+18}$;

$x_{p+12 n+10}>x_{p+12 n+8}>x_{p+12 n+6}$;

(iii) $x_{p+12 n+23}>x_{p+12 n+20}>x_{p+12 n+17}$;

$x_{p+12 n+11}>x_{p+12 n+8}>x_{p+12 n+5}$.

Also, the following inequalities hold.

(iv) $x_{p+12 n+18} x_{p+12 n+13}>1$ and $x_{p+12 n+13} x_{p+12 n+11}<1$;

(v) $x_{p+12 n+17} x_{p+12 n+14}>1$ and $x_{p+12 n+14} x_{p+12 n+11}<1$.

In fact, from the observations

$$
\begin{aligned}
& x_{p+12 n+18} \\
& =\frac{x_{p+12 n+16} x_{p+12 n+15}+x_{p+12 n+16} x_{p+12 n+13}+x_{p+12 n+15} x_{p+12 n+13}+1+a}{x_{p+12 n+16} x_{p+12 n+15} x_{p+12 n+13}+x_{p+12 n+16}+x_{p+12 n+15}+x_{p+12 n+13}+a} \\
& >\frac{1}{x_{p+12 n+13}}
\end{aligned}
$$

and

$$
\begin{aligned}
& x_{p+12 n+13} \\
& =\frac{x_{p+12 n+11} x_{p+12 n+10}+x_{p+12 n+11} x_{p+12 n+8}+x_{p+12 n+10} x_{p+12 n+8}+1+a}{x_{p+12 n+11} x_{p+12 n+10} x_{p+12 n+8}+x_{p+12 n+11}+x_{p+12 n+10}+x_{p+12 n+8}+a} \\
& <\frac{1}{x_{p+12 n+11}},
\end{aligned}
$$

we know that the two inequalities in (iv) are true. The proofs for two inequalities of $(\mathrm{v})$ are similar.

Combining the above inequalities (ii), (iii), (iv) and (v), one can derive that

$$
\begin{aligned}
& x_{p+12 n+22}>x_{p+12 n+20}>x_{p+12 n+18} \\
& >\frac{1}{x_{p+12 n+13}} \\
& >x_{p+12 n+10}>x_{p+12 n+8}>x_{p+12 n+6}
\end{aligned}
$$

and

$$
\begin{aligned}
& x_{p+12 n+23}>x_{p+12 n+20}>x_{p+12 n+17} \\
& >\frac{1}{x_{p+12 n+14}} \\
& >x_{p+12 n+11}>x_{p+12 n+8}>x_{p+12 n+5} .
\end{aligned}
$$

(i) shows that the sequences $\left\{x_{p+12 n}\right\}_{n=0}^{\infty},\left\{x_{p+12 n+2}\right\}_{n=0}^{\infty}$, $\left\{x_{p+12 n+4}\right\}_{n=0}^{\infty},\left\{x_{p+12 n+7}\right\}_{n=0}^{\infty}$ and $\left\{x_{p+12 n+9}\right\}_{n=0}^{\infty}$ are decreasing with lower bound 1. Therefore, their limits exist, are finite and are equal. That is,

$$
\begin{aligned}
& \lim _{n \rightarrow \infty} x_{p+12 n}=\lim _{n \rightarrow \infty} x_{p+12 n+2} \\
& =\lim _{n \rightarrow \infty} x_{p+12 n+4}=\lim _{n \rightarrow \infty} x_{p+12 n+7} \\
& =\lim _{n \rightarrow \infty} x_{p+12 n+9}=L .
\end{aligned}
$$

(6) and (7) display that the sequences $\left\{x_{p+12 n+5}\right\}_{n=0}^{\infty}$, $\left\{x_{p+12 n+6}\right\}_{n=0}^{\infty},\left\{x_{p+12 n+8}\right\}_{n=0}^{\infty},\left\{x_{p+12 n+10}\right\}_{n=0}^{\infty}$ and $\left\{x_{p+12 n+11}\right\}_{n=0}^{\infty}$ are increasing with upper bound 1 . Therefore, their limits exist and are finite, moreover are equal. That is,

$$
\begin{aligned}
& \lim _{n \rightarrow \infty} x_{p+12 n+5}=\lim _{n \rightarrow \infty} x_{p+12 n+6} \\
& =\lim _{n \rightarrow \infty} x_{p+12 n+8}=\lim _{n \rightarrow \infty} x_{p+12 n+10} \\
& =\lim _{n \rightarrow \infty} x_{p+12 n+11}=M .
\end{aligned}
$$

Furthermore, (6) and (7) indicate 
$L=\lim _{n \rightarrow \infty} x_{p+12 n+2}=\lim _{n \rightarrow \infty} x_{p+12 n+14}$

$=\frac{1}{M}=\lim _{n \rightarrow \infty} x_{p+12 n+13}=\lim _{n \rightarrow \infty} x_{p+12 n+1}$.

Now, we prove that $L=M=1$. To this end, noting that

$x_{p+12 n+11}$

$=\frac{x_{p+12 n+9} x_{p+12 n+8}+x_{p+12 n+9} x_{p+12 n+6}+x_{p+12 n+8} x_{p+12 n+6}+1+a}{x_{p+12 n+9} x_{p+12 n+8} x_{p+12 n+6}+x_{p+12 n+9}+x_{p+12 n+8}+x_{p+12 n+6}+a}$

and taking the limit on both sides of the above equality, one can see that

$M=\frac{L M+L M+M M+1+a}{L M M+L+M+M+a}$.

Solving this equation with (8), one can see $L=M=1$. From $1<x_{p+12 n+3}<x_{p+12 n+1}$ and $\lim _{n \rightarrow \infty} x_{p+12 n+1}=1$, it is easy to obtain $\lim _{n \rightarrow \infty} x_{p+12 n+3}=1$. Up to this, we have shown $\lim _{n \rightarrow \infty} x_{p+12 n+k}=1, k=0,1, \cdots, 11$, which says that (5) holds in the case $(\mathrm{C} 1)$.

Now, we deal with Case (C3). Similar to Case (C1), for some nonnegative integer $p$, we denote by $\left\{x_{p}, x_{p+1}, x_{p+2}\right\}^{+}$ the terms of a positive semicycle of length three, followed by $\left\{x_{p+3}\right\}^{-}$a negative semicycle with length one and so on.

Hence, the rule for the positive and negative semicycles to occur successively can be periodically expressed as follows:

$\left\{x_{p+4 n}, x_{p+4 n+1}, x_{p+4 n+2}\right\}^{+},\left\{x_{p+4 n+3}\right\}^{-}, n=0,1, \cdots$.

Still according to Lemma 2.2 (b), (c) and (d), we have

$x_{p+4 n+4}<x_{p+4 n+2}<x_{p+4 n}$ and $x_{p+4 n+8}<x_{p+4 n+5}<x_{p+4 n}$. (9)

Similar to the derivation of (iv) in Case (C1), we also obtain

$$
x_{p+4 n+5}<\frac{1}{x_{p+4 n+3}}<x_{p+4 n+1} .
$$

It follows from (9) that $\left\{x_{p+4 n}\right\}_{n=-4}^{\infty}$ is decreasing with lower bound 1 . Therefore, its limit exists and is finite. Moreover, it is also clear from (9) that

$\lim _{n \rightarrow \infty} x_{p+4 n}=\lim _{n \rightarrow \infty} x_{p+4 n+2}=\lim _{n \rightarrow \infty} x_{p+4 n+5}=L$.
Accordingly, we obtain from (10) that $\lim _{n \rightarrow \infty} x_{p+4 n+3}=1 / L$.

Now, we prove that $L=1$. To this end, noting that

$x_{p+4 n+5}$

$=\frac{x_{p+4 n+3} x_{p+4 n+2}+x_{p+4 n+3} x_{p+4 n}+x_{p+4 n+2} x_{p+4 n}+1+a}{x_{p+4 n+3} x_{p+4 n+2} x_{p+4 n}+x_{p+4 n+3}+x_{p+4 n+2}+x_{p+4 n}+a}$

and taking the limit on both sides of the above equality, we get

$L=\frac{1 / L L+1 / L L+L L+1+a}{1 / L L L+1 / L+L+L+a}$.

Solving this equation produces $L=1$. Hence, $\lim _{n \rightarrow \infty} x_{p+4 n+k}=1, k=0,1,2,3$, which says that (5) holds in the case (C3). Thus, the proof of Theorem 3.3 is complete.

\section{REFERENCES}

[1] Agarwal RP. Difference equations and inequalities, Marcel Dekker, New York, 1992 (1st edition); 2000 (2nd edition).

[2] Kocic VL, Ladas G. Global behavior of nonlinear difference equations of higher order with applications, Kluwer Academic Publishers, Dordrecht 1993.

[3] Kulenović MRS, Ladas G, Martins LF, Rodrigues IW. The Dynamics of $x_{n+1}=\frac{\alpha+\beta x_{n}}{A+B x_{n}+C x_{n-1}}$ : facts and conjectures. Comput Math Appl 2003; 45: 1087-99.

[4] Patula WT, Voulov HD. On the oscillation and periodic character of a third order rational difference equation. Proc Am Math Soc 2002; 131 (3): 905-9.

[5] Camouzis E, DeVault R, Papaschinopoulos G. On the recursive sequence. Adv Differ Equa 2005; 1 (2005); 31-40.

[6] Li X. The rule of semicycle and global asymptotic stability for a fourth-order rational difference equation. Comput Math Appl 2005; 49(5/6): 723-30

[7] Li X, Zhu D. Global asymptotic stability for two recursive difference equations. Math Appl Comput 2004; 150 (2): 481-92.

[8] Li X, Zhu D. Global asymptotic stability of a nonlinear recursive sequence. Math Appl Lett 2004; 17 (7): 833-38.

[9] Li X. Qualitative properties for a fourth order rational difference equation. J Math Anal Appl 2005; 311(1): 103-11.

[10] $\mathrm{Li} \mathrm{X}$. The rule of trajectory structure and global asymptotic stability for a nonlinear difference equa, Math Appl Lett 2006; 19: $1152-58$

This is an open access article distributed under the terms of the Creative Commons Attribution License (http://creativecommons.org/licenses/by/2.5/), which permits unrestrictive use, distribution, and reproduction in any medium, provided the original work is properly cited. 